\title{
Cervical cancer patients presentation and survival in the only oncology referral hospital, Ethiopia: a retrospective cohort study
}

Muluken Gizaw ${ }^{1,2^{*}}$ (D), Adamu Addissie ${ }^{2}$, Sefonias Getachew ${ }^{1,2}$, Wondimu Ayele ${ }^{1,2}$, Israel Mitiku ${ }^{4}$, Ulrike Moelle ${ }^{5}$, Tigist Yusuf ${ }^{2}$, Mathias Begoihn ${ }^{5}$, Mathewos Assefa ${ }^{6}$, Ahmedin Jemal ${ }^{3}$ and Eva Johanna Kantelhardt ${ }^{1,5}$

\begin{abstract}
Background: Women infected with Human Immune Deficiency Virus (HIV) are assumed to be at higher risk of developing Cervical Cancer (CC). This is due to a rapid progression of pre-invasive to invasive lesions. However, evidences suggest, due to the availability of antiretroviral therapy (ART) and care services; an improved survival and treatment outcome of CC patients (CCPS) with HIV infection is expected.

Objective: The aim of this study is to examine the clinical characteristics and survival of of CCPs registered at the radiotherapy center of Tikur Anbessa Specialized Hospital (TASH), Addis Ababa University, Ethiopia.

Methods: We conducted a retrospective cohort study. Data from 1655 CCPs diagnosed between September 2008 and September 2012 were included. The primary endpoint was death from any cause. Kaplan-Meier estimates were compared using the log-rank test. Cox proportional hazards regression model was used to identify predictors of death. Data were analyzed using STATA version IC/14.

Results: The mean age of all patients was 49 years ( $S D=11.6$ years). Of all CCPs, 139 (8.4\%) were HIV positive, 372 (22. 5\%) patients had a known negative HIV status and 1144 (69.1\%) patients were asymptomatic with unknown HIV status. Due to late stage and waiting times, only $13.5 \%$ of the patients received curative radiotherapy doses. HIV-positive CCPs presented more often with advanced disease compared to HIV negative CCPs ((44.6\%) versus $39.7 \%, p=0.007)$. There was no significant difference in survival between HIV-positive and HIV-negative CCPs. Older age (HR=2.01; 95\% Cl, 1. 01,-4.05), advanced disease $(H R=2.6 ; 95 \% \mathrm{Cl}, 1.67-4.04)$ and baseline anemia $(H R=1.65 ; 95 \% \mathrm{Cl}, 1.24,2.20)$ were independent predictors for higher risk of death.
\end{abstract}

Conclusion: Survival rates of CCPs did not differ according to HIV status. The risk of death was higher for patients with older age, advanced disease and anemia. HIV patients should be screened for CC according to guidelines to avoid late presentation.

Keywords: Uterine cervical neoplasms, HIV, Survival, Africa, Ethiopia

\footnotetext{
* Correspondence: muluken.gizaw@yahoo.com

${ }^{1}$ Institute of Medical Epidemiology, Biostatistics and Informatics,

Martin-Luther-University, Halle (Saale), Germany

${ }^{2}$ Department of Preventive Medicine, School of Public Health, Addis Ababa

University, Addis Ababa, Ethiopia

Full list of author information is available at the end of the article
} 


\section{Background}

Cancer and other non-communicable diseases (NCDs) have become leading causes of disability and death in developing countries, including Ethiopia [1]. Cervical Cancer (CC) is a leading cause of cancer morbidity and mortality in women globally. In 2012, 528, 000 new cases and 270,000 deaths were estimated to have occurred worldwide, with the majority of these cases and deaths (90\%) occurring in low- and middle-income countries [2]. In Ethiopia, CC is the second most commonly diagnosed cancer and the leading cause of cancer death in women, with about 8000 newly diagnosed cases and 4700 deaths every year [3]. Most CC patients in Ethiopia seek healthcare at an advanced stage, when the effectiveness of treatment is limited [4].

Women infected with HIV are presumed to be more likely to have high risk Human Papilloma Virus (HPV) and have at least a $10 \%$ higher risk of developing CC [5-8]. HIV-positive patients are reported to more likely present with advanced stages of CC. It has been shown that HIV changes the natural history of HPV infection, resulting in a rapid progression to invasive lesions, and are associated with adverse survival probabilities [9]. The overall HIV prevalence in adult population of Ethiopia was $1.18 \%$ in 2016 with highest prevalence in Addis Ababa and Gambela regions with 4.9 and $4 \%$ respectively [10].

However, in the current context, due to the availability of ART and care services, an improved survival and treatment outcome of cervical cancer patients with HIV infection is expected. There is no adequate information documenting this evidence in Ethiopia. Hence, we conducted a retrospective cohort study to assess the survival rate of cervical cancer patients according to HIV status. We reviewed 1655 charts of women with cervical cancer from Tikur Anbessa University Hospital in Addis Ababa, Ethiopia.

\section{Methods}

\section{Study design and population}

We conducted a retrospective cohort study among cervical cancer patients diagnosed at Tikur Anbessa (Black lion) Specialized Hospital (TASH) from September 2008 to September 2012. TASH is the national teaching and referral hospital with more than 800 beds and offers diagnosis and treatment for approximately 400,000 inpatients and out-patients a year. The hospital receives patients who are referred from across the country, as well as patients from Addis Ababa. The hospital is the only one with a radiotherapy facility in Ethiopia. Patients were treated with surgery in the early stages and according to locally adapted guidelines at the radiotherapy center. Brachytherapy was not available at the time. Patients also may have registered but there after not received any treatment. Demographic and clinical characteristics of the patients were retrieved from individual patient charts. The survival status of patients was collected from the cancer registry which obtained information from patient cards or via telephone calls,

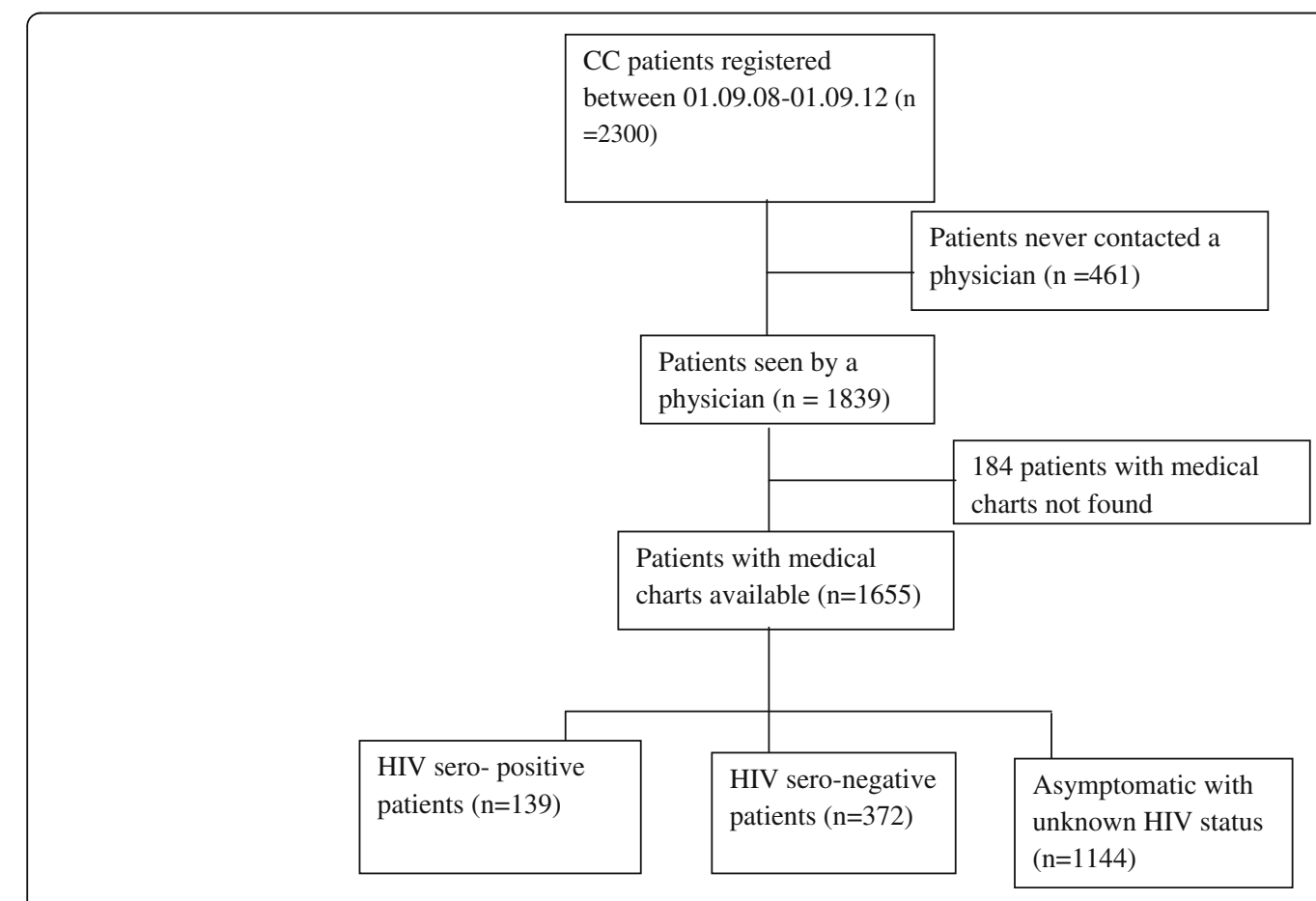

Fig. 1 Total number of cervical cancer Patients from radiotherapy center at TASH, Addis Ababa, Ethiopia, included in the final analysis (Strobe diagram) 


\section{Study variables and data collection HIV status identification}

The HIV status of each patient was retrieved from medical charts by trained medical staff. Only half of the patient's charts contained a registered HIV status. HIV status was documented if the patient had been screened for HIV. Since September 10, 2011 every patient registered at TASH had been screened for HIV on a regular basis. Before this, only patients with a high risk profile (e.g., HIV-positive partner) or clinically suspicious patients were screened. The HIV status was tested using the enzyme-linked immunosorbent assay method. We grouped HIV status into three categories: HIV-positive, HIV-negative and HIV unknown.

\section{Study outcome and definition}

The primary objective of this study was to compare the overall survival of CC patients according to HIV status. We estimated follow-up time between the date of first presentation and the last date of observation. The last date of observation was defined as either death or censoring at the last known alive.

\section{Data analysis}

STATA version IC/14 (StataCorp, College Station, TX, USA) was used for statistical analyses. The overall survival of HIV positive and negative patients was estimated using Kaplan-Meier methods. Kaplan-Meier estimates were compared using the log-rank test. Cox proportional hazards regression model was used to identify predictors for survival. Influences of prognostic factors were estimated using hazard ratios with 95\% confidence intervals (CI).

\section{Ethical considerations}

Ethical approval was obtained from the institutional review board of the College of Health Sciences, Addis Ababa University and Martin Luther University, Halle Germany. The confidentiality of the patient status was maintained by avoiding personal identifiers during analysis.

\section{Results}

Of the 1839 cervical cancer patients registered and seen by physicians at TASH, medical charts were retrieved for 1655 (90.0\%). Of the 1655 patients with a medical chart, 139 (8.4\%) were HIV-positive, 372 (22.5\%) were HIVnegative and 1144 (69.1\%) were asymptomatic with unknown HIV status (see Fig. 1).

Of all patients, 1081(65.3\%) patients received any form of radiotherapy, 190 (11.5\%) underwent surgery and 206 (12.4\%) received chemotherapy. Among patients treated by radiotherapy, non- radical radiotherapy was provided for $770(71.0 \%)$ of stage IIIB and IVA patients. Of the 190 patients who underwent surgery, 155 (81\%) received
Table 1 Demographic and clinical characteristic of cervical cancer patients according to HIV status, TASH, Addis Ababa, Ethiopia, 2008-2012

\begin{tabular}{|c|c|c|c|}
\hline \multirow{2}{*}{$\begin{array}{l}\text { Patient demographic } \\
\text { characteristics }\end{array}$} & \multicolumn{3}{|l|}{ HIV status } \\
\hline & $\begin{array}{l}\text { HIV negative } \\
\mathrm{n}(\%)\end{array}$ & $\begin{array}{l}\text { HIV positive } \\
\text { n (\%) }\end{array}$ & $\begin{array}{l}\text { HIV unknown } \\
\mathrm{n}(\%)\end{array}$ \\
\hline \multicolumn{4}{|l|}{ Residence } \\
\hline Urban & $128(34.4)$ & $82(59)$ & $431(37.7)$ \\
\hline Rural & $244(65.6)$ & $57(41)$ & $713(62.3)$ \\
\hline \multicolumn{4}{|l|}{ Marital status } \\
\hline Single & $3(0.8)$ & $5(3.6)$ & $4(0.4)$ \\
\hline Married & $297(79.8)$ & $113(81.3)$ & $928(81.1)$ \\
\hline Unknown & $72(19.4)$ & $21(15.1)$ & $212(18.5)$ \\
\hline \multicolumn{4}{|l|}{ Age category } \\
\hline$<30$ & $15(4.0)$ & $29(20.9)$ & $47(4.1)$ \\
\hline $30-39$ & $81(21.8)$ & $58(41.7)$ & $243(21.2)$ \\
\hline $40-49$ & $131(35.2)$ & $40(28.8)$ & $389(34)$ \\
\hline $50-59$ & $88(23.7)$ & $10(7.2)$ & $295(25.8)$ \\
\hline $60+$ & $57(15.3)$ & $2(1.4)$ & $170(14.9)$ \\
\hline \multicolumn{4}{|c|}{ FIGO stage at presentation } \\
\hline H-IA & $59(15.8)$ & $10(7.2)$ & $95(8.3)$ \\
\hline$\|B-\| A$ & $148(39.8)$ & $57(41.0)$ & $51(45.1)$ \\
\hline IIIB-IVA & $144(38.7)$ & $62(44.6)$ & $458(40.0)$ \\
\hline IVB & $6(1.6)$ & $5(3.6)$ & $15(1.3)$ \\
\hline Post-operative & $7(1.9)$ & $2(1.4)$ & $23(2.0)$ \\
\hline Recurrence & $7(1.9)$ & $3(2.2)$ & $28(2.5)$ \\
\hline Unknown & $1(0.3)$ & 0 & $9(0.8)$ \\
\hline \multicolumn{4}{|l|}{ Anemia Status } \\
\hline No anemia $\geq 12$ & $186(50)$ & $51(36.7)$ & $437(38.2)$ \\
\hline$>10$ and $<12$ & $93(25)$ & $38(27.3)$ & $373(32.6)$ \\
\hline $8-10$ & $36(9.7)$ & $24(17.3)$ & $153(13.4)$ \\
\hline$>5$ and $<8$ & $24(6.5)$ & $12(8.6)$ & $73(6.4)$ \\
\hline$<5$ & $15(4.0)$ & $12(8.6)$ & $60(5.2)$ \\
\hline Unknown & $18(4.9)$ & $2(1.4)$ & $48(4.2)$ \\
\hline \multicolumn{4}{|l|}{ Co-morbidity status } \\
\hline No co morbidity & 341 (91.7) & $124(89.2)$ & 1045(91.3) \\
\hline Any co morbidity & $31(8.3)$ & $15(10.8)$ & $99(8.7)$ \\
\hline \multicolumn{4}{|l|}{ Treatment modalities } \\
\hline Radiation & $187(63.8)$ & $105(79.5)$ & $789(75.0)$ \\
\hline Surgery & $71(24.2)$ & $12(9.0)$ & $107(10.0)$ \\
\hline Chemotherapy & $35(12.0)$ & $15(11.5)$ & $156(15.0)$ \\
\hline \multicolumn{4}{|l|}{ Patient outcome } \\
\hline Alive & $331(89.0)$ & $108(77.7)$ & $930(81.3)$ \\
\hline Dead & $41(11.0)$ & $31(22.3)$ & $214(18.7)$ \\
\hline Total & 372 & 139 & 1144 \\
\hline
\end{tabular}


radical hysterectomy and nine received a simple hysterectomy (see Table 1).

\section{Patient characteristics}

Table 1 shows the demographic and clinical characteristics of patients according to their HIV status. The mean age of all patients at entry was 49 years. The majority of cervical cancer patients with HIV-positive status came from urban areas (59\%), while the majority of patients with HIVnegative, HIV unknown status were rural residents (62 and $66 \%$, respectively). The majority of HIV-positive cervical cancer patients were between 30 and 39 years old $(42 \%)$, with mean age of 39 ( $\mathrm{SD}=9)$ whereas HIV negative, HIV unknown cervical cancer patients were between 40 and 49 years (35 and 34\%, with mean age of 50, respectively). About $81 \%$ of cervical cancer patients were married. The International Federation of Gynecology and Obstetrics (FIGO) stage at presentation for HIV-positive patients was IIB-IIIA (41\%) and IIIB-IVA (44.6\%); for HIV-negative/unknown patients IIB-IIIA (40 and 45\%, respectively) and IIIB-IVA (39 and 40\%, respectively). A total of $120(86.3 \%)$ of HIV positive women were on ART.

\section{Survival according to HIV status}

A total of 286 (17.3\%) cervical cancer patients died during the follow-up period. The median survival time was 38 months.. Of the total deaths, $41(11 \%)$ and $31(22.3 \%)$ were HIV negative and HIV Positives. The median survival of HIV positives and HIV negative was 29 and 28 months respectively.

Crude survival probabilities did not differ between patients according to HIV status. After adjusting for place of residence, age, FIGO stage, co-morbidity (yes/ no) and baseline anemia status, no difference in survival probability was seen between HIV-positive and HIVnegative/unknown cervical cancer patients $(H R=1.16$, 95\%CI 0.70-1.91) (see Fig. 2).

\section{Survival according to other clinical and demographic characteristics}

After adjusting for place of residence, HIV status, FIGO stage, co-morbidity and baseline anemia status, older CC patients had a two-fold higher risk of death than younger patients $(\mathrm{HR}=2.02,95 \% \mathrm{CI}: 1.01-4.05)$. CC patients with higher cancer stage (FIGO IIB-IIIA and recurrence) had a higher risk of death, with $\mathrm{HR}=2.60(95 \% \mathrm{CI}$ : $1.67-4.04)$ and HR $=2.77$ (95\%CI: 1.35-5.68), respectively, compared to those with a lower stage. Cervical cancer patients with anemia at baseline were more likely to die than nonanemic patients with $\mathrm{HR}=1.65$ (95\%CI: 1.24-2.20) and $\mathrm{HR}=1.84(95 \% \mathrm{CI}: 1.27-2.66)$ for a hemoglobin level of greater than 10 and between 8 and 10, respectively. Place of residence and co-morbidity status did not show any differences in overall survival of CC patients (see Table 2).

\section{Discussion}

This study showed that CC patients with known HIV infection constituted $8.4 \%$ of all $\mathrm{CC}$ patients registered in the largest referral hospital in Ethiopia. The survival rate was similar among CC patients with and without known HIV infection in this cohort where only $14 \%$ of patients received any form of therapy considering only the curative radiotherapy. The majority of patients with known HIV infection came from the urban area, compared to patients without HIV infection. About $86 \%$ of the HIV positive CCPs were on ART. A slightly higher proportion of patients with HIV infection presented with late-

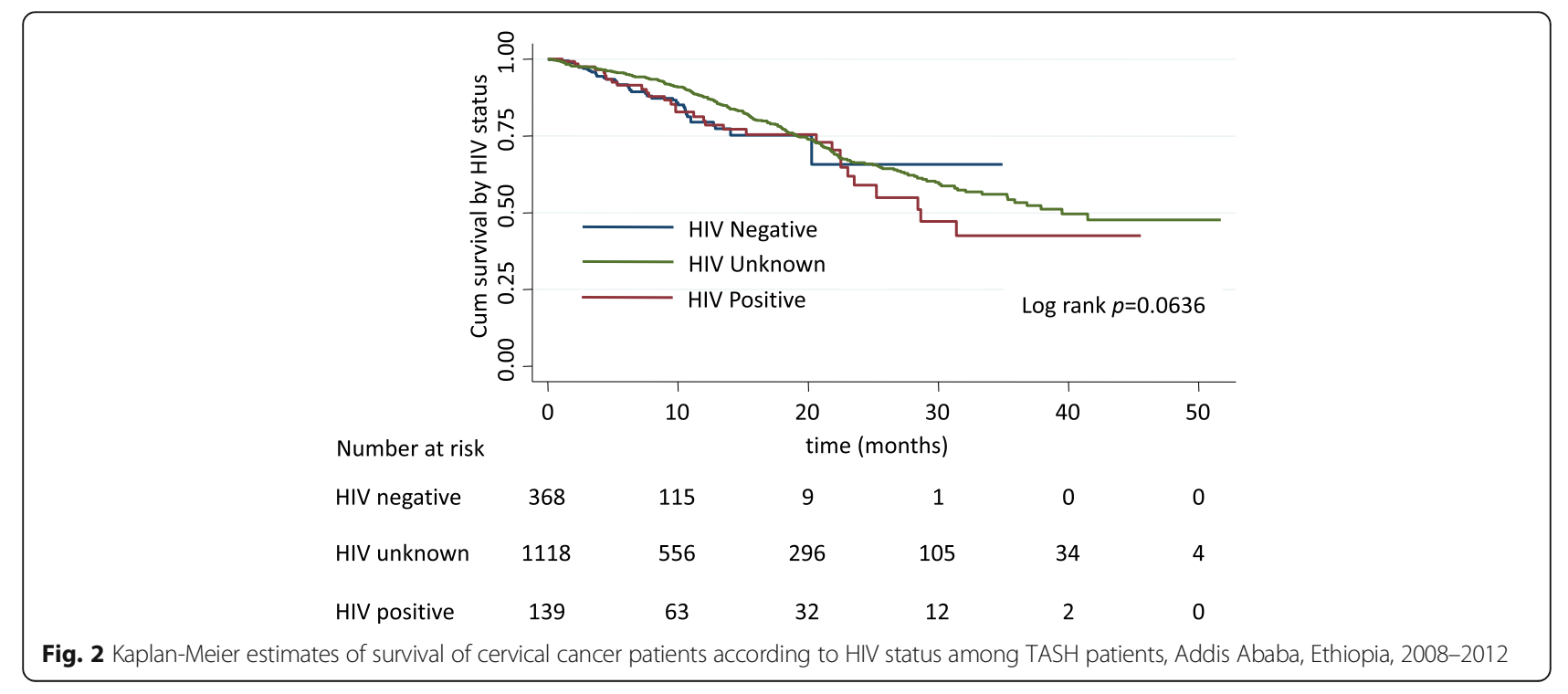


Table 2 Demographic and clinical characteristic associated with the survival of cervical cancer patients, TASH, Addis Ababa, Ethiopia, 2008-2012

\begin{tabular}{|c|c|c|c|}
\hline Characteristics & Unadjusted HR (95\% Cl) & Adjusted HR (95\% Cl) & $P$-value \\
\hline \multicolumn{4}{|l|}{ HIV status } \\
\hline Positive & 1.00 & 1.00 & \\
\hline Negative & $1.13(0.70,1.81)$ & $1.16(0.70,1.91)$ & 0.564 \\
\hline Unknown & $0.77(0.53,1.13)$ & $0.76(0.51,1.15)$ & 0.206 \\
\hline \multicolumn{4}{|l|}{ Residence } \\
\hline Rural & $1.2(0.94,1.51)$ & $1.16(0.91,1.48)$ & 0.212 \\
\hline Urban & 1.00 & 1.00 & \\
\hline \multicolumn{4}{|l|}{ Age group } \\
\hline$<30$ & 1.00 & 1.00 & \\
\hline $30-39$ & $1.52(0.78,2.97)$ & $1.43(0.73,2.82)$ & 0.290 \\
\hline $40-49$ & $1.50(0.78,2.86)$ & $1.47(0.75,2.87)$ & 0.259 \\
\hline $50-59$ & $1.12(0.57,2.20)$ & $1.17(0.58,2.36)$ & 0.650 \\
\hline $60+$ & $1.83(0.93,3.61)$ & $2.01(1.01,4.05)^{a}$ & 0.049 \\
\hline \multicolumn{4}{|c|}{ FIGO stage at presentation } \\
\hline$|-| \mid A$ & 1.00 & 1.00 & \\
\hline$\|\mathrm{B}-\| \mathrm{A}$ & $1.32(0.86,2.03)$ & $1.22(0.79,1.88)$ & 0.372 \\
\hline IIIB-IVA & $3.07(2.00,4.71)$ & $2.60(1.67,4.04)^{a}$ & $<0.001$ \\
\hline IVB & $3.77(1.13,12.55)$ & $2.54(0.74,8.68)$ & 0.137 \\
\hline Post-operative & $1.17(0.45,3.07)$ & $1.04(0.40,2.74)$ & 0.930 \\
\hline Recurrence & $2.46(1.21,5.00)$ & $2.77(1.35,5.68)^{a}$ & 0.005 \\
\hline \multicolumn{4}{|c|}{ Anemia: Hgb level at presentation (g/dl) } \\
\hline No anemia $\geq 12$ & 1.00 & 1.00 & \\
\hline$>10$ and $<12$ & $1.82(1.38,2.40)$ & $1.65(1.24,2.20)^{a}$ & 0.001 \\
\hline $8-10$ & $2.20(1.54,3.14)$ & $1.84(1.27,2.66)^{\mathrm{a}}$ & 0.001 \\
\hline$<8$ and $\geq 5$ & $1.47(0.90,2.42)$ & $1.35(0.81,2.25)$ & 0.242 \\
\hline$<5$ & $1.24(0.67,2.31)$ & $1.12(0.60,2.10)$ & 0.706 \\
\hline Unknown & $1.74(0.23,12.73)$ & $1.21(0.16,8.96)$ & 0.846 \\
\hline \multicolumn{4}{|l|}{ Co-morbidity status } \\
\hline No co-morbidity & 1.00 & 1.00 & \\
\hline Any co-morbidity & $0.98(0.68,1.40)$ & $0.99(0.68,1.43)$ & 0.969 \\
\hline
\end{tabular}

HR hazard ratio, Cl confidence interval, HIV Human Immune deficiency Virus, FIGO International Federation of Gynecology and Obstetrics values in boldface are statistically significant at alpha of 0.05

stage cancer. The median time of observation in patients without event was 38 months. Older age, late-stage disease and anemia were factors significantly influencing overall mortality probabilities of cervical cancer patients.

The survival of CC patients with positive HIV status was similar to those with negative or asymptomatic with unknown HIV status. The observed survival of HIV positive patients could have been compromised for two reasons: either because they die due to the HIV infection or second because the CC is more aggressive. Since we do not have information on cause of death in our study, we can only suggest that the widespread use of ART in this cohort may prevent HIV related deaths and also fast progression of CC [11-13].

A higher proportion of HIV-infected cervical cancer patients presented with advanced stages of cancer compared to those with negative/unknown HIV status. This is probably because the HIV infection decreases the progression time of cervical cancer to more advanced stages $[9,14]$.

In our Cox model, we found older age, baseline anemia and advanced stage to be significantly associated with higher all-cause mortality of cervical cancer patients. This finding is consistent with studies conducted 
in similar settings elsewhere. According to a Nigerian study, there was a $41 \%$ higher proportion of death for advanced stages compared to early stages [15]. Moreover, a Kenyan finding showed that the 2-year survival of cervical cancer patients at advanced stage was less than $20 \%[16]$.

Baseline anemia independently predicted a higher risk of death; moderate anemia was significantly associated with higher mortality compared to patients with no anemia. In this study, anemia was defined as a hemoglobin level below $12 \mathrm{~g} / \mathrm{dl}$. Another similar study from north-central Nigeria indicated baseline anemia to be an independent predictor of lower survival in cervical cancer patients [15]. Furthermore, older ( $\geq 60$ years) patients had a significantly higher risk of death compared to younger patients. Several other studies have also reported this $[17,18]$; this might be due to fact that young patients are more likely to respond to treatment and present in an early stage [17]. In addition, it has to be considered that the probability for all-cause death is higher in the older age groups.

The strength of this study is the large number of all CC patients with medical charts available during a 4-year period in TASH, the only hospital for cancer treatment in Ethiopia and the inclusion of those patients who only registered but never received treatment were included. However, a limitation is the large proportion of CC patients with only asymptomatic with unknown HIV status. Since their characteristics were very similar to those with negative HIV sero-status we assume they are more likely to be HIV sero-negative. However, this may have underestimated the effect of HIV on the survival of CC patients. Moreover, about $20 \%$ of all cervical cancer patients who registered at TASH and were scheduled to see a physician for treatment planning during the study period did not come back; these patients may have had very advanced disease. We assume that the $10 \%$ of charts missing were random cases due to problems of misplacing, or miss-spelling names or numbers. The huge efforts that would have been needed to retrieve these charts were out of scale.

\section{Conclusion}

In conclusion, known HIV-positive patients constitute a considerable proportion of CC patients in a hospital cohort in Ethiopia and are diagnosed at a more advanced stage of disease compared to those with negative and unknown status. Survival did not differ between HIVpositive and HIV-negative and -unknown CC patients after adjusting for other prognostic factors. The high proportion of advanced stage cancer in HIV-positive patients suggests the need to increase the implementation and awareness of cervical cancer screening among HIVpositive women and remove barriers to accessing screening. Anemia at presentation probably reflects the severity of disease and therefore shows adverse survival. Attention should be given generally to those $\mathrm{CC}$ patients who are diagnosed at older age and with advanced stage of disease, as these are contributing factors for lower survival rates. Finally, a high proportion of unknown HIV status justifies screening for HIV in all CC patients, this is now in place.

\section{Abbreviations \\ ART: Antiretroviral Therapy; CC: Cervical Cancer; CCPs: Cervical Cancer Patients; Cl: Confidence Intervals; FIGO: International Federation of Gynecology and Obstetrics; HIV: Human Immune Deficiency Virus; HPV: Human Papilloma Virus; HR: Hazard Ratio; NCDs: Non-communicable Diseases; SD: Standard Devation; TASH: Tikur Anbessa Specialized Hospital}

\section{Acknowledgements}

Authors would like to thank the staff members of Tikur Anbessa Specialized Hospital Radiotherapy Center who treated and provided care for all cervical cancer patients. We also grateful to Mrs. Tinsae and Mrs. Tigist for obtaining the follow-up data and Mrs. Mulu and Mr. Neme for collecting the patient files.

\section{Funding}

Funding for this research was provided by the Federal Ministry of Research and Education of Germany, grant number 01DG12006 and 01DG13026. This work was also supported by the German Ministry for Economic and Development Cooperation (BMZ) through the ESTHER University and Hospital Partnership Initiative of German International Cooperation (GIZ).

\section{Availability of data and materials}

- The datasets analyzed are available from the corresponding author on reasonable request.

\section{Authors' contributions}

MG performed statistical analysis and draft the manuscript. EK and AA participated in designing the study, analysis, reviewing and editing the final manuscript and contributed to the discussion. UM, TY and MB carried out the conception, designing the study and data collection. SG, WA, IM, MA and AJ have been involved in revising the manuscript critically with valid inputs. All authors read and approved the final form of the manuscript.

Ethics approval and consent to participate

Ethical approval was obtained from the institutional review board of the College of Health Sciences, Addis Ababa University and Martin Luther University, Halle Germany.

Consent for publication

Not Applicable.

Competing interests

The authors declare that they have no competing interests.

\section{Publisher's Note}

Springer Nature remains neutral with regard to jurisdictional claims in published maps and institutional affiliations.

\section{Author details}

${ }^{1}$ Institute of Medical Epidemiology, Biostatistics and Informatics, Martin-Luther-University, Halle (Saale), Germany. ${ }^{2}$ Department of Preventive Medicine, School of Public Health, Addis Ababa University, Addis Ababa, Ethiopia. ${ }^{3}$ Department of Intramural Research, American Cancer Society, Atlanta, GA, USA. ${ }^{4}$ Department of Public Health, College of Medicine and Health Sciences, Wollo University, Dessie, Ethiopia. ${ }^{5}$ Department of Gynecology, Martin-Luther-University, Halle (Saale), Germany. ${ }^{6}$ Radiotherapy Center, School of Medicine, Addis Ababa University, Addis Ababa, Ethiopia. 
Received: 21 September 2017 Accepted: 23 November 2017

Published online: 29 November 2017

\section{References}

1. Lozano R, Naghavi M, Foreman K, et al. Global and regional mortality from 235 causes of death for 20 age groups in 1990 and 2010: a systematic analysis for the global burden of disease study 2010. Lancet (London, England). 2012;380(9859):2095-128.

2. Torre LA, Bray F, Siegel RL, et al. Global cancer statistics, 2012. CA Cancer J Clin. 2015;65(2):87-108.

3. ICO. Human Papilloma Virus and related cancers, fact sheet; 2016. Available from: URL: http://www.hpvcentre.net/statistics/reports/ETH_FS.pdf. Cited 14 July 2016.

4. Kantelhardt EJ, Moelle U, Begoinn M, et al. Cervical cancer in Ethiopia: survival of 1,059 patients who received oncologic therapy. Oncologist. 2014; 19(7):727-34.

5. Denslow SA, Rositch AF, Firnhaber C, et al. Incidence and progression of cervical lesions in women with HIV: a systematic global review. Int J STD AIDS. 2014;25(3):163-77.

6. WHO. Guidelines for screening and treatment of precancerous lesions for cervical cancer prevention: 2013.

7. Vuyst $\mathrm{H}$, Mugo NR, Chung $\mathrm{MH}$, et al. Prevalence and determinants of human papillomavirus infection and cervical lesions in HIV-positive women in Kenya. Br J Cancer. 2012;107(9):1624-30.

8. Denny $L$, Boa R, Williamson AL, et al. Human papillomavirus infection and cervical disease in human immunodeficiency virus-1-infected women. Obstet Gynecol. 2008;111(6):1380-7.

9. Clarke B. Postmodern cancer: the role of human immunodeficiency virus in uterine cervical cancer. Mol Pathol. 2002;55(1):19-24.

10. The Ethiopian Public Health Institute (EPHI). HIV related estimates and projections for Ethiopia-2017; 2017. Available from: URL: https://www.ephi. gov.et/images/pictures/download2009/HIV_estimation_and_projection_for_ Ethiopia_2017.pdf. Cited 15 Nov 2017.

11. Bonnet F, Burty C, Lewden C, et al. Changes in cancer mortality among HIVinfected patients: the mortalité 2005 survey. Clin Infect Dis. 2009;48(5):633-9.

12. Collaborative analysis of 13 HIV cohort studies. Causes of death in HIV-1infected patients treated with antiretroviral therapy, 1996-2006. Clin Infect Dis. 2010;50(10):1387-96.

13. Gotti D, Raffetti $E$, Albini $L$, et al. Survival in HIV-infected patients after a cancer diagnosis in the CART Era: results of an Italian multicenter study. PLoS One. 2014;9(4):e94768.

14. Mbulaiteye SM, Bhatia K, Adebamowo C, et al. HIV and cancer in Africa: mutual collaboration between HIV and cancer programs may provide timely research and public health data. Infect Agent Cancer. 2011;6(1):16

15. Musa J, Nankat J, Achenbach CJ, et al. Cervical cancer survival in a resourcelimited setting-North Central Nigeria. Infect Agent Cancer. 2016;11:15.

16. Maranga IO, Hampson L, Oliver AW, et al. Analysis of factors contributing to the low survival of cervical cancer patients undergoing radiotherapy in Kenya. PLoS One. 2013;8(10):e78411.

17. Zhou J, Li X, Huang K, et al. Young cervical cancer patients may be more responsive than older patients to neoadjuvant chemotherapy followed by radical surgery. PLoS One. 2016;11(2):e0149534.

18. Dryden P, Scott B, Suneja G, et al. HIV infection and survival among women with cervical cancer. JCO. 2016;34(31):3749-57.

\section{Submit your next manuscript to BioMed Central and we will help you at every step:}

- We accept pre-submission inquiries

- Our selector tool helps you to find the most relevant journal

- We provide round the clock customer support

- Convenient online submission

- Thorough peer review

- Inclusion in PubMed and all major indexing services

- Maximum visibility for your research

Submit your manuscript at www.biomedcentral.com/submit 